Website: ijetms.in Issue:5, Volume No.4, September-2020 DOI: 10.46647/ijetms.2020.v04i05.007

\title{
INTERNET OF THINGS (IOT): HOW DID IT START, WHATS ITS PRESENT STATE -A RESEARCH NOTION
}

\author{
Mrs.Preeti C.M. \\ Lecturer, Dept of CSE, \\ Loyola Academy, Alwal, \\ Hyderabad,India. \\ preeti.gadasu28@gmail.com
}

\author{
Dr.T. Santhi Sri, \\ Associate Professor, \\ Dept of CSE, KLEF, Vaddeswaram, \\ Guntur, India. \\ santhisri@kluniversity.in
}

\begin{abstract}
In developing applications for the Internet of Things, lot of interest is being developed from the kids to the old ones.The main goal is to integrate technology into the world. The implementation is discussed here regarding integration. This paper describes the initiation form the basic communication mode to various applicationsin architectural presentation of how world is operating and how people are integrated with the IoT. The results of integration are the way to new ways of scenarios in IoT that support evolution to the Internet of People. This paper discusses abouthow research is carried in general.
\end{abstract}

\section{Keywords: IoT; IoE;Communication;IoP; IoT Platform}

\section{INTRODUCTION}

The method of moving data from a region, people or a group to anyone is called communication. Communication includes a sender,recipient and message.These include our feelings, the cultural activities, the wayof communication, and the location.Communication started with telegraph,landline telephone,radio and tv, fax,mobile telephone and finally computers and internet [4]. The present world waits for the result to be executed in a few seconds when a query is requested by them. This state of mind has bought into existence the IOT. Inshort, the IoT is called as, growth of the Internet whereall objects have network connection, that make them to send and receive data. Communication applications in different ways which include document transfer, long way communication, voicecommunication and modern communication [4]. Its components include sender, receiver, transmission medium, protocols and message. The goal lineis applicationdevelopment for the IoTand to integrate technology into everybody'slife. It shows how this integration is implemented, and leaves scope for growth. The user setslimitations in the application, and when the person changes framework, they should manually organize. Here,description ofastructure that uses smartphones to improve integration with the IoT, leading its path to 
Website: ijetms.in Issue:5, Volume No.4, September-2020 DOI: 10.46647/ijetms.2020.v04i05.007

new IoT situations that support growth towards the IoP.

\section{LITERATURE SURVEY}

IoT combines many things in the world [1]. It can't act independently. Computers,Humans, smart objects are bought together to form a network. Thepresentnetwork systems arecloud computing, social network, along withInternet andsmart phones. The diversednetworks [3] have bought the concepts ofCoT-Cloud of Things, WoT Web of Things and SIoT - Social Internet of Things.IoT connectsvaried objects in lifewhich operate withtransceivers and microcontrollersfor communicating digitally. Many devices like homeneeds, cameras, sensors which are used for monitoring interact withIOT toenable easy and smart works. There are varieties of devices connected to IOT, here we face the problem ofheterogeneous. Diverse isdefined in IOTthatlink to different types of networks, varied kinds of data that are gathered from different devices and the technology that is used for single device.

\section{A. Network}

A computer network is defined as collection of computer equipments that are connected with wires, optical fibers, wireless links, the various separate devices are called nodes thatcan "talk" to each another and exchangeinformation.A network analysis starts with a notion that the primary business starts by studying of the social structure[2]. This social structure analyzes people's actions based on structural constraints. It grabs attention of seeing the world that uses different bounds in hierarchical structures. A direct way to know about the social structure is to analyze the patterns that includes its members. A network analyst searches for deep patterns that includes regular network patterns.

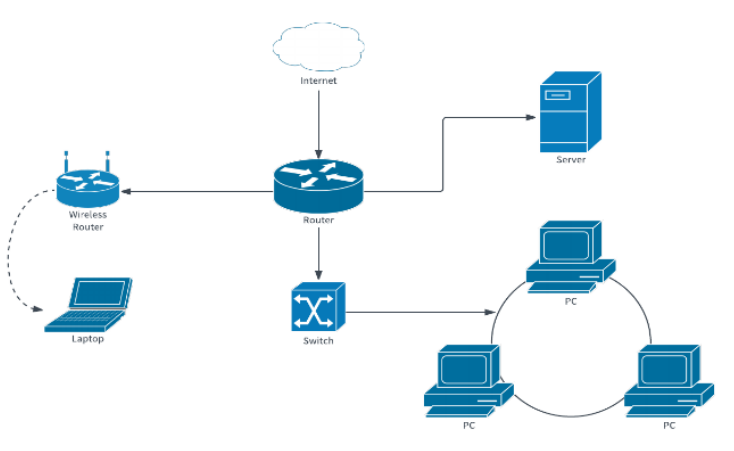

Fig 1: Network

Theydescribe the patterns and implement the descriptions to knowabout how the network structure limits social behavior. On studying the structural properties of networks, it informs how-to carry-on research, organize data collection and develop analytic methods.Few applications include terminal emulation, file transfer, email programs, resource sharing, video sharing, chatting.A router is a networking device that moves data packets between computer networks [2]. Routers task is to check the traffic guiding functions on the world wide web. Data that is sent through the internet,like a web page, email, is a kind of the data packets. A switching network is a kind ofnetworking hardware that links devices on a computer network that uses packet switching to receive and move data to the destination. A network attendant is a computer system, used as centralsource of data and different programs which are shared by different people in anetwork. The applications of the internet go like sending and receiving emails, searching and browsing data, copying files between computers, making financial transactions, navigating in the car, a smart bike and many more like , 
Website: ijetms.in Issue:5, Volume No.4, September-2020 DOI: 10.46647/ijetms.2020.v04i05.007

playing interactive games, streaming audio, video, chatting and video conferencing.

\section{B. Internetof Things}

Internet of Things (IoT)deals with anetworking objects with internet andcollectsdata and exchanges information.A modest way thethings that sense,gather data, and then sends it to the internet. The physical things embedded with actuators, sensors, and other electronics. Its features include connect, control, monitor, exchange and sense. To initiate communication, connects one device to IoT platform [7]. It uses actuators to achieve automation and then control electrical appliances. The exchange of data among different IoT gadgets, IoT enabled platform to store data is also maintained. By using sensors, sensing the change of varied physical quantities like temperature, light intensity humidity. Finally, the question arises why move towards IOT.The various factors like monitor, improvement, save, enhance, integrate and decision made everyone move towards IoT. It monitors overall business processes, improves customer experience, saves money, time and other resources, enhances employee productivity, integrates and adapts business models, helps in making better business decisions. The increase in productivity, minimizing human effort, enhancing asset utilization, improve safety and security are few positive aspects. Few negative aspects like security, complexity and privacy are observed in IoT.

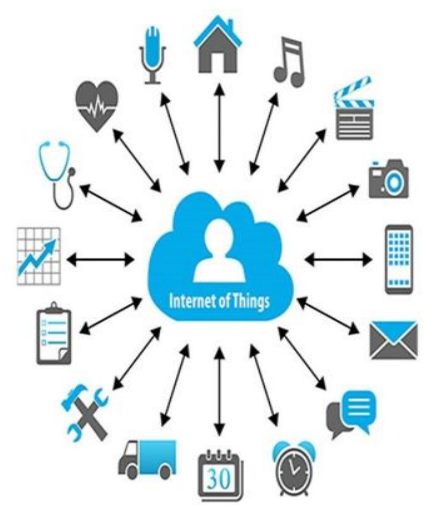

Fig2: Internet of Things[5]

\section{Internet Of People}

Theavailability of internet is almost everywhere havinga myriad of devices equipped with a connecting interface. Linking everyphysical object to the internet is getting easier, thus making Internet of Things (IoT) popular. The goal of smart integrating the devices is to simplify everyone's lives by making technology work for all. For instance, each one can remotely switch on our house air conditioning using smartphone to get a required temperature before we reach home.IoT technologies are currently integrated with humans where there is lot of space for improvement. The technology has to develop relevant mechanisms to adapt to people'sintention. When we consider an IoT scenario, technologytakes people's context into account, learn from them, and take steps according to the situation and expectation, that avoids user intervention. Therefore, if we plan to arrive home late, there would be like the AC kept off till they're actually on their way back home.To enable some scenarios like these, it requires us to move from the Internet of Things to the Internet of People [8](IoP).

\section{Internet of Everything}

The Internet of Everything (IoE) isto bring people together, processdata, 
Website: ijetms.in Issue:5, Volume No.4, September-2020 DOI: 10.46647/ijetms.2020.v04i05.007

and things, so as to make networked connections relevant and valuable like ever before-turning data into actions that make new capabilities, richer experiences, and unprecedented economic opportunities.

\section{E. Layers of IoT}

There is a fast growth in technology today, it'sdifficult to keep track of the rise of different technologies. But there is a common point of the modern technology, that is technology convergence and Internet of things is the best example. The convergence of different technologies works together to solve a real business problem or to obtain new products and services[9]. In this paper, link from IOT, cloud, Big Data, Business value is discussed.

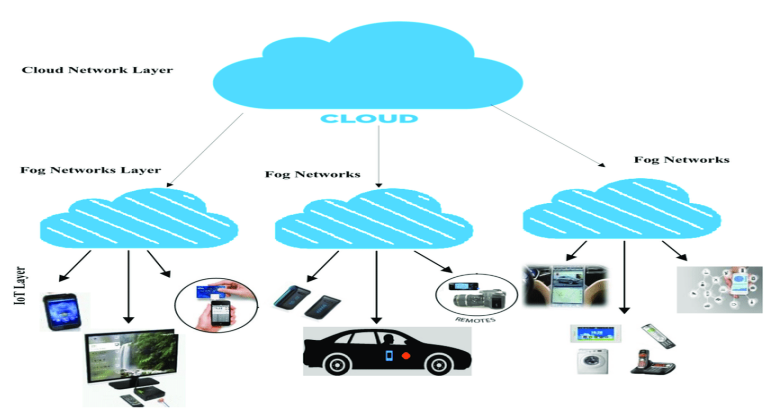

Fig3: Layers of IoT[10]

\section{1) Fog Layer}

Fog layer is of a distributed network environment and is closely associated with cloud computing and the internet of things (IoT). Fog computing is a distributed network which connects these two environments. In this computing, a decentralized computing infrastructure has data that computes, stores and the applications are located between the data and the cloud. To build this layer, we need devices, sensors, controllers that lead to communications, machine to machine communications(M2M), networks, protocols, Wi-Fi connections, hardware components.

\section{2) Cloud Layer}

Computing with the cloudhinges on common computing dataand not havingpersonal devices to manage requests. The amenities are carried out and are used with the Internet and paid by the cloud client whenneeded and pay for a business type structure. The community cloud is stated as computing services given by providers over the Internet, by making available to all who want to buy them. They may be free or sold onneeded basis, allowing customers to pay per usage for the CPU cycles, storage, or bandwidth they use.A private cloud service is not shared with any other organization. In contrast, a public cloud service that shares computing services with different customers, though each customer's data and applications that run in the cloud remain hidden from other cloud customers. A hybrid cloud is an infrastructure which includes links between one cloud that is managed by the user and at least one cloud is managed by a third party.

\section{3) Big Data}

Big Data can be software-utility that is designed to analyse, process and extract thedata from a complex and large data sets. Big data describes the large volume of data, that is structured and unstructured that has a business on a day-to-day basis. Big data analyseson insights that lead to better decisions and strategic business. Bigdata Analytics uses Machine Learning algorithms. ML algorithms are involved in the process to train thetechnologies by giving the datasets andusethealgorithms that make machines workin problem-solving and decision making.

\section{4) Business Value}


Website: ijetms.in Issue:5, Volume No.4, September-2020 DOI: 10.46647/ijetms.2020.v04i05.007

Custom application development is

the process of designingthe software applications for a specific user or group of usersin an organization. This custom software is designed to address specific needs in an organization.

\section{ARCHITECTURE of IOT}

In IoT system, [11] connected devices are responsible for providing the data. To choose the physical parameters in the outside world orin the object itself,we need sensors. Theyare embedded in the devicesor they caneither beimplemented by standalone objects to note the measure and get data. As an example, let's think of an agricultural related sensor whoseworkis measuring parameters like air, soil temperature, humidity, soil with $\mathrm{pH}$ levels and crop exposed to the sunlight.The essentials of the layer include the actuators. As in close association with sensors, they change the data produced by smart objects into the physical action. Basingupon the inputsgiven by the sensors, the system inspects the situation in real life,orders the actuators to expose theparticular water valves that aresituated in locations where the soil moisture is below to the set value. The valves remain open till the sensors state that the values are returned to default. This can happen without human involvement.

\section{A. Sensors}

A sensor discovers the change in the environment and sends the data to theelectronics [6].Itcan be a device, component, machine,subsystem whose work is to find events, related changes in environment and move the data to different electronics, like a computer workstation. Theoften-used sensors are divided based upon the measureslike Electric current, Potential,Magnetic, Humidity sensor,
Fluid velocity sensor, Flow control sensors, Pressure related sensors, Thermal based sensors, Radio sensors, Proximity sensors, Optical sensors, Position sensors. A sensor changes stimulus such as heat, light, sound, motion into electrical signals. Thesignals are passed through an interface which converts them into a binary code and passes this on to a computer to be processed. In easy terms, it senses organs of the device used to detect changes in the environment.

\section{B. Actuators}

An actuator[12] is a component of a machine that helps in moving and controlling a mechanism, for example by opening a valve. In simple terminology it is a mover. An actuator needs a control signal and a source of energy. An actuatorfunctions to provide thrust and positioning in machines that is used for testing. One form is the electromechanical actuator, that converts the torque of an electric rotary motor into linear mechanical thrust. For a mechanical device, an actuator is a component that converts the control signal intomovement. Examples are Electric motors. Solenoids. Hard drive stepper motors. In other words it acts as hands of a device used for controlling. 
Website: ijetms.in Issue:5, Volume No.4, September-2020 DOI: 10.46647/ijetms.2020.v04i05.007
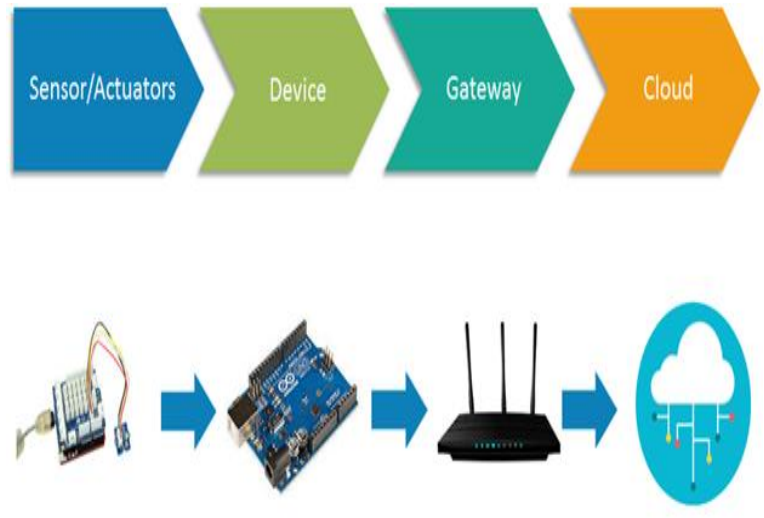

Fig4: Actuators[12]

\section{Processor}

A processor processes all on an IoT device, it works like the brain of any device. It is used to get the readings of the sensorsand handle user inputs. It Involves in controlling actuators.A processor communicates with the modules that communicates.

\section{Connectivity}

The connection can be enabled in this manner;

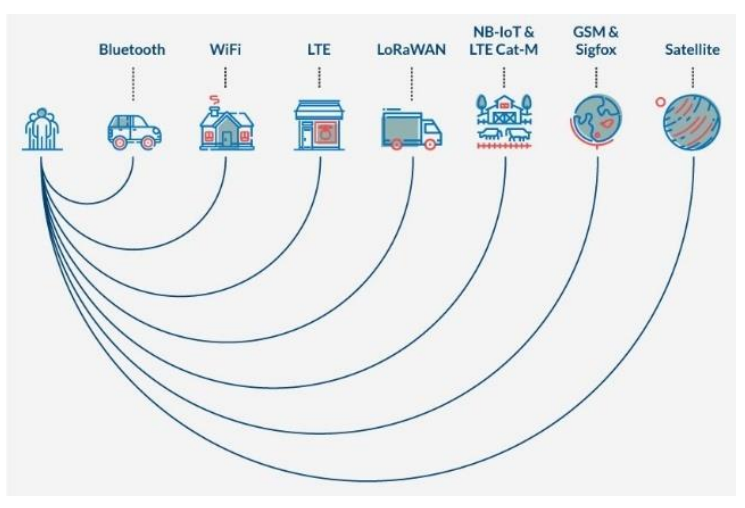

Fig5: Connection

\section{E. Gateway}

The IoT gateway is a mainfeature in IoT ecosystems. It acts as the bridge of communications. It links Zigbee sensors with the Internet, Bluetooth devices with a cloud application. It helps reduce latency and improve capacity at the edge by preprocessing raw data.An IoT gateway bridges the communication gap between sensors, equipment, devices, systems, andcloud. On systematically connecting to the cloud, IoT gateway makes local processing and storage, asan ability to autonomously control field devices that are based on data inputs by the sensors.

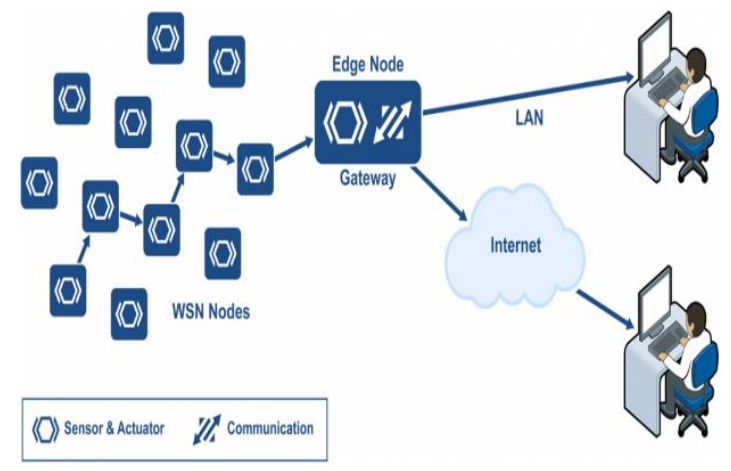

Fig6: IoT Gateway

\section{F. IoT platform}

An IoT platform is a multi-layer technology that makes straightforward provisioning, management, automation of connected devicesin the Internet of Things. IoT is a non-standard field. For creating a proper IoT platform, there are many ways. We can use an Arduino or raspberry piwith some sensors and use some commercial, open source tools to provide a gateway interface and see information in the platform.IoT platforms helps Connect hardware, like sensors and devices to handle different hardware and software communication protocols. This provides security and authentication for devices and users. It Collects, visualizes, and analyse data that the sensors and devices collect. 
Website: ijetms.in Issue:5, Volume No.4, September-2020 DOI: 10.46647/ijetms.2020.v04i05.007

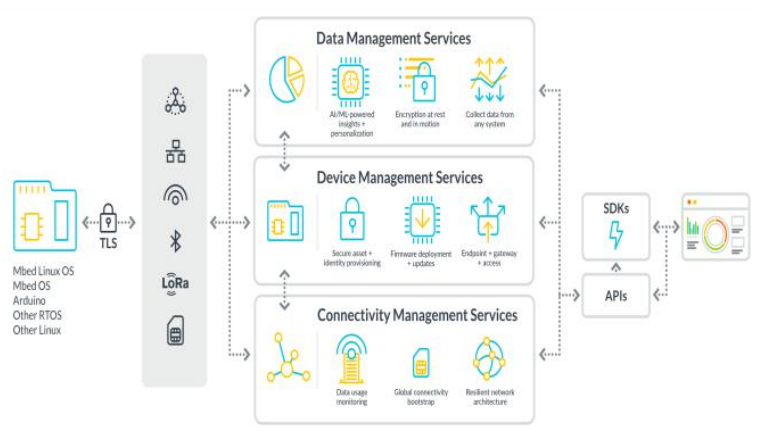

Fig7: IoT Platform

\section{CONCLUSION}

The basic idea of presenting this paper is to have an idea of how to proceed with research in Internet of Things (IoT) that is changing the world. The research can be carried out on any internal areas of layers discussed or the architecture, so that analysing the data and bringing out new methods.

\section{REFERENCE}

[1]https://www.researchgate.net/publicatio n/334636380_A_Literature_Survey_on_In ternet_of_Things_security issues\#: : text= In\%20day\%2Dtoday\%20life\%2C\%20IoT, for\%20the\%20changes $\% 20 \mathrm{in} \% 20$ environ ment.

[2]L.Atzori, A. Iera, and G. Morabito, "The internet of things" A survey, Comput.Netw, 2010, vol. 54, no. 15, pp. 2787-2805.

[3] Ke $\mathrm{Xu}, \mathrm{Yi} \mathrm{Qu}$, andKun Yang. "A Tutorial on the Internet of Things:From Heterogeneous Network Integration Perspective" IEEE network,March/April 2016.

\section{[4] Communication} www.skillsyouneed.com > ips , what-iscommunication

[5]https://www.google.com/url?sa=i\&url= https\%3A\%2F\%2Fwww.cybersecurityinsi ders.com\%2Fdemystifying-network- isolation-and-micro-

segmentation $2 \% 2 \mathrm{~F} \& \mathrm{psig}=\mathrm{AOvVaw} 0 \mathrm{Hu} 7 \mathrm{~J}$

SoGRclkWTkMmsoC49\&ust=159085683

$0533000 \&$ source $=$ images $\& c d=$ vfe $\& v e d=0$

CAIQjRxqGAoTCNCspNnB2ekCFQAA

AAAdAAAAABC6Ag

[6] https://en.wikipedia.org/wiki/Sensor

[7] https://www.google.com/search?q=inter net+of+things\&rlz=1C1SQJL_enIN902IN 902\&sxsrf=ALeKk02vO1kF_YNblg2Z4D $\underline{\mathrm{icH}}$

kbwdFepg:1591984325715\&source=lnms \&tbm=isch\&sa $=X \& v e d=2 a h U K E w i f o O P h$ 6_zpAhVxyDgGHez6CF8Q_AUoAnoEC $\underline{B w Q B A \& b i w}=1536 \&$ bih $=754 \#$ imgrc $=A 1$ 9MPd2TxTBWwM

[8] https://www.researchgate.net/publication/2 73792989 From the Internet of Things to the Internet of People

[9]https://analyticstraining.com/4-layersof-the-internet-of-things/

[10]https://www.google.com/url?sa=i\&url $=$ https $\% 3 \mathrm{~A} \% 2 \mathrm{~F} \% 2 \mathrm{Fwww}$. researchgate.net \%2Ffigure\%2FProposed-5G-enabled-FogComputing-and-Internet-of-Things-IoTbased-

Architecture_fig1_324603012\&psig=AOv Vaw1WtF3609J_y-

DM7xyK9YwN\&ust $=1592069298603000$ \&source $=$ images \&cd $=v f e \& v e d=0 C A I Q j R$ xqFwoTCICu9MHm_OkCFQAAAAAdA AAAABAQ

[11]https://www.avsystem.com/blog/whatis-iot-architecture/

[12]https://www.google.com/url?sa=i\&url $=$ https $\% 3 \mathrm{~A} \% 2 \mathrm{~F} \% 2 \mathrm{Fwww}$.javatpoint.com $\% 2$ Fiot-

architecturemodels\&psig=AOvVaw2es7_5 Kw_6g_u5lxF_Wkun\&ust $=159207044159$ 9000\&source $=$ images $\& c d=$ fe $\& v e d=0 C A$ IQjRxqFwoTCMj47eTq_OkCFQAAAAA dAAAAABAm 
International Journal of Engineering Technology and Management Sciences[IJETMS]

Website: ijetms.in Issue:5, Volume No.4, September-2020 DOI: 10.46647/ijetms.2020.v04i05.007 